\title{
Hypoxic renal injury in newborns with abdominal compartment syndrome (clinical and experimental study)
}

\author{
Dmitry Morozov ${ }^{1}$, Olga Morozova ${ }^{2}$, Dmitri Pervouchine ${ }^{3}$, Lubov Severgina ${ }^{4}$, Alexei Tsyplakov ${ }^{5}$, Natalya Zakharova ${ }^{5}$, \\ Nikita Sushentsev ${ }^{2}$, Larisa Maltseva ${ }^{2}$ and Ivan Budnik ${ }^{2}$
}

BACKGROUND: Surgical treatment for gastroschisis and congenital diaphragmatic hernia $(\mathrm{CDH})$ commonly leads to abdominal compartment syndrome (ACS) associated with hypoxic renal injury. We hypothesized that measurement of urinary and serum concentrations of vascular endothelial growth factor (VEGF), $\pi$-glutathione S-transferase ( $\pi-G S T)$, and monocyte chemoattractant protein-1 (MCP-1) may serve for noninvasive detection of hypoxic renal injury in such patients. METHODS: Intra-abdominal pressure (IAP), renal excretory function, and the biomarker levels were analyzed before, 4, and 10 days after surgery. Association between the biomarker levels and renal histology was investigated using an original model of ACS in newborn rats.

RESULTS: Four days after surgery, IAP increased, renal excretory function decreased, and the levels of VEGF, $\pi-G S T$, and MCP-1 increased, indicating renal injury. Ten days after surgery, IAP partially decreased, renal excretory function completely restored, but the biomarker levels remained elevated, suggesting the ongoing kidney injury. In the model of ACS, increase in the biomarker levels was associated with progressing kidney morphological alteration.

CONCLUSION: Surgical treatment for gastroschisis and $\mathrm{CDH}$ is associated with prolonged hypoxic kidney injury despite complete restoration of renal excretory function. Follow-up measurement of VEGF, $\pi-G S T$, and MCP-1 levels may provide a better tool for noninvasive assessment of renal parenchyma in newborns with ACS.

I ntra-abdominal hypertension (IAH) is defined as a sustained or repeated pathological elevation in intraabdominal pressure (IAP) $\geq 12 \mathrm{~mm} \mathrm{Hg}$ (1). IAH with sustained IAP above $20 \mathrm{~mm} \mathrm{Hg}$ may lead to development of abdominal compartment syndrome (ACS) characterized by a significant reduction in abdominal perfusion pressure causing organ dysfunction/failure and ultimately death, if untreated $(1,2)$. Constantly updated clinical guidelines developed by the World Society of the Abdominal Compartment Syndrome
(WSACS) have helped to reduce ACS mortality in adults $(2,3)$. However, in newborns, it is still high, reaching $60 \%$ $(4,5)$. Neonates with specific birth defects associated with visceral-abdominal disproportion, including gastroschisis and congenital diaphragmatic hernia $(\mathrm{CDH})$, are at the highest risk of developing ACS after surgical treatment (6).

ACS is commonly associated with renal hypoperfusion, circulatory hypoxia, and subsequent renal injury. Injury to the renal tissue induces local inflammatory response that may result in renal scarring and chronic kidney disease later on (7-9). However, diagnostic techniques for detection and monitoring of hypoxic renal injury in newborns with ACS are still lacking. Previous studies have demonstrated that the measurement of serum and urinary levels of some biomarkers may serve as a useful tool for noninvasive assessment of renal parenchyma. It has been shown that vascular endothelial growth factor (VEGF) levels correlate with the severity of renal hypoxia (10), $\pi$-glutathione $S$-transferase ( $\pi$-GST) levels correlate with distal tubule injury (11), and monocyte chemoattractant protein-1 (MCP-1) levels indicate the extent of the inflammatory cell infiltration in the kidney (12). Therefore, in the present study, we hypothesized that concentrations of VEGF, $\pi$-GST, and MCP- 1 may serve as potential biomarkers of hypoxic renal injury in newborns with ACS. To examine this possibility, we measured serum and urinary concentrations of these biomarkers before and after surgical treatment in children with gastroschisis and $\mathrm{CDH}$, and using an original model of ACS in newborn rats, we investigated whether the changes in the biomarker levels were associated with kidney morphological alterations resulting from IAH.

\section{METHODS}

Ethics

The study was approved by the Institutional Review Board. All children were included in the study after their parents agreed and signed the informed consent form. Care and handling of the animals were in accord with the National Institutes of Health guidelines.

\footnotetext{
${ }^{1}$ Department of Pediatric Surgery, Sechenov First Moscow State Medical University, Moscow, Russia; ${ }^{2}$ Department of Pathophysiology, Sechenov First Moscow State Medical University, Moscow, Russia; ${ }^{3}$ Center for Data-Intensive Biomedicine and Biotechnology, Skolkovo Institute of Science and Technology, Moscow, Russia; ${ }^{4}$ Department of Pathological Anatomy, Sechenov First Moscow State Medical University, Moscow, Russia; ${ }^{5}$ Research Institute for Fundamental and Clinical Uronephrology, Saratov State Medical University n. a. V. I. Razumovsky, Saratov, Russia. Correspondence: Ivan Budnik, (budnik.ivan@gmail.com) 


\section{Patients}

A total of 27 newborns admitted for surgical treatment of uncomplicated gastroschisis $(n=14)$ and $\mathrm{CDH} \quad(n=13)$ were prospectively enrolled in this study between January 2014 and June 2015. The exclusion criteria were: presence of additional birth defects, severe cerebral ischemia, infection, and systemic inflammatory disease. Birth defects were diagnosed antenatally on routine ultrasound scanning. The control group included 20 newborns admitted for surgical treatment of minor omphalocele. The results of all clinical, instrumental, and laboratory data, including IAP, renal RI, urine output, serum creatinine, and the biomarkers, were collected at three time points of the study: on the day of admission (before surgery), on POD4, and on POD10.

\section{IAP Measurement}

IAP was measured by intravesical manometry as described previously (13). The urinary bladder was emptied, and sterile normal

Table 1. Characteristics of healthy controls and patients with congenital defects

\begin{tabular}{lccc}
\hline Variable & $\begin{array}{c}\text { Control } \\
(n=20)\end{array}$ & $\begin{array}{c}\text { Gastroschisis } \\
(n=14)\end{array}$ & $\begin{array}{c}\mathrm{CDH} \\
(n=13)\end{array}$ \\
\hline $\begin{array}{l}\text { Age at repair } \\
\text { (days) }\end{array}$ & $10.4 \pm 3.1$ & $1.4 \pm 0.5$ & $2.7 \pm 1.2$ \\
$\begin{array}{l}\text { Sex, male, } n(\%) \\
\text { Birth weight (g) }\end{array}$ & $12(60 \%)$ & $8(57 \%)$ & $7(54 \%)$ \\
$\begin{array}{l}\text { Gestation } \\
\text { (weeks) }\end{array}$ & $38 \pm 1.5$ & $2,164 \pm 325$ & $2,650 \pm 461$ \\
Apgar score & $8 \pm 2$ & $31 \pm 1.7$ & $37 \pm 1.8$ \\
\hline
\end{tabular}

$\mathrm{CDH}$, congenital diaphragmatic hernia.

The values shown are mean \pm SD. saline was instilled into the bladder with a volume of $1 \mathrm{ml} / \mathrm{kg}$. The feeding tube was held vertically up and the height of the column of saline from midaxillary line at the level of iliac crest was measured at the end of expiration. IAP was measured in $\mathrm{cmH}_{2} \mathrm{O}$ and converted to $\mathrm{mm} \mathrm{Hg}\left(1 \mathrm{~mm} \mathrm{Hg}=1.36 \mathrm{cmH}_{2} \mathrm{O}\right)$.

\section{Renal RI Measurement}

Renal Doppler ultrasound examinations were performed on the interlobar or arcuate arteries using 3.5 to $5.5 \mathrm{MHz}$ transducer. The Doppler spectrum was considered optimal when three consecutive similar-appearing waveforms were obtained. The renal RI was calculated as follows: (peak systolic velocity - end diastolic velocity)/ peak systolic velocity. Three measurements were performed on each kidney and averaged to obtain the mean RI value.

\section{Model of ACS in Newborn Rats}

A total of 100 newborn Wistar rats (age range, 2-6 h) were equally randomized into five groups. Group 1 served as a control (mean IAP, $2 \mathrm{~mm} \mathrm{Hg}$ ). Groups 2 and 3 were exposed to mild IAH (mean IAP, $9 \mathrm{~mm} \mathrm{Hg}$ ) for 3 and 8 days, respectively. Groups 4 and 5 were exposed to severe IAH (mean IAP, $17 \mathrm{~mm} \mathrm{Hg}$ ) for 3 and 8 days, respectively. Rats were anesthetized with isoflurane-oxygen mixture and subjected to urinary catheterization and intravesical manometry using $24 \mathrm{G}$ peripheral venous catheter. IAP was measured in $\mathrm{cmH}_{2} \mathrm{O}$ and converted to $\mathrm{mm} \mathrm{Hg}$. IAH was created by intraperitoneal injection of crosslinked bovine collagen (Zyplast; Allergan, Irvine, CA) under control of intravesical manometry. In the control group, the abdominal cavity was pierced without further injection of the collagen gel.

\section{Histological Examination}

Kidneys were obtained from each killed rat, fixed in 10\% neturalbuffered formalin, and subjected to routine tissue processing.
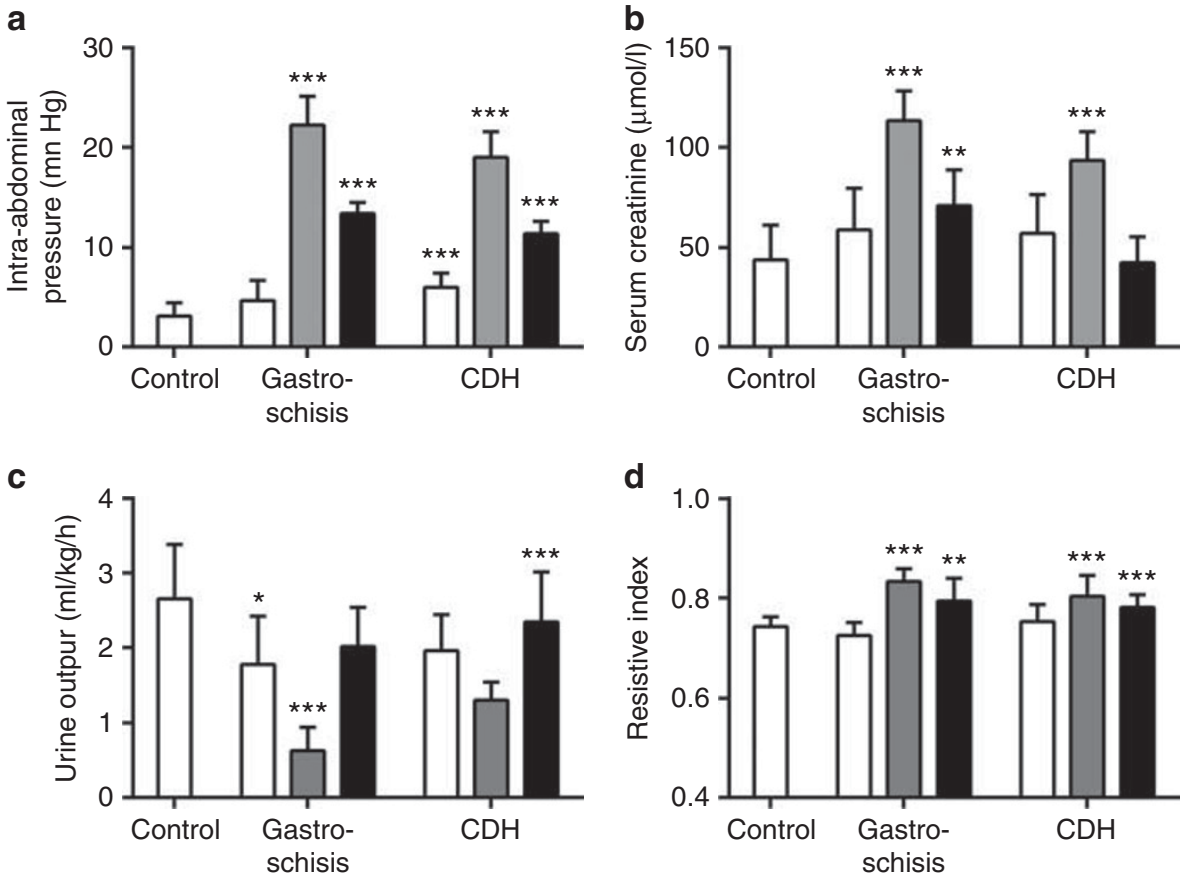

Figure 1. Laboratory and instrumental data in controls and patients with gastroschisis and congenital diaphragmatic hernia (CDH). (a) Intraabdominal pressure; (b) serum creatinine; (c) urine output; (d) resistive index. The data are presented as mean \pm SD. White bars denote admission values, grey bars postoperative day 4 (POD4) values, and black bars POD10 values. The data were analyzed using one-way analysis of variance (ANOVA), followed by a post hoc $t$-test corrected for multiple hypothesis testing. Comparison vs. control: ${ }^{*} P<0.05 ;{ }^{* *} P<0.01 ;{ }^{* * *} P<0.001$. 

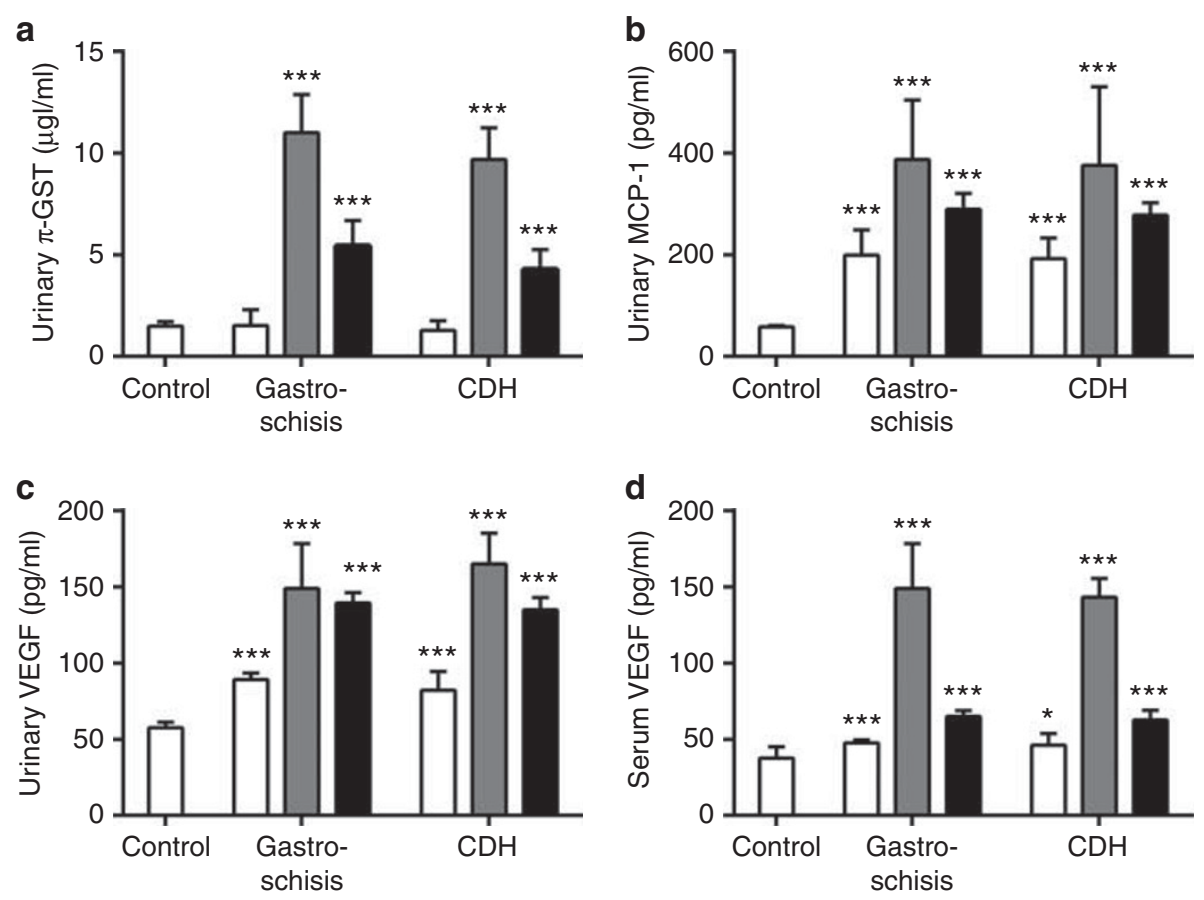

Figure 2. The biomarker levels in controls and patients with gastroschisis and congenital diaphragmatic hernia (CDH). (a) Urinary $\pi-g l u t a t h i o n e$ S-transferase ( $\pi$-GST); (b) urinary monocyte chemoattractant protein-1 (MCP-1); (c) urinary vascular endothelial growth factor (VEGF); (d) serum VEGF. The data are presented as mean \pm SD. White bars denote admission values, grey bars postoperative day 4 (POD4) values, and black bars POD10 values. The data were analyzed using one-way analysis of variance (ANOVA), followed by a post hoc $t$-test corrected for multiple hypothesis testing. Comparison vs. control: ${ }^{*} P<0.05 ;{ }^{* *} P<0.01 ;{ }^{* * *} P<0.001$.

Five-micrometer-thick sections were prepared, stained with hematoxylin and eosin, and examined by light microscopy.

\section{Biomarker Measurement}

In patients, blood samples were obtained through a central venous catheter, and urine samples were obtained by urinary catheterization. In experimental animals, blood was collected by cardiac puncture under anesthesia. Blood and urine samples were centrifuged at $3,000 \mathrm{~g}$ for $15 \mathrm{~min}$, and supernatants were immediately stored at $-25^{\circ} \mathrm{C}$ for no more than 1 month until testing. Concentrations of VEGF, $\pi$-GST, and MCP-1 were measured by enzyme-linked immunosorbent assay. All specimens were measured in duplicate wells, and the mean of the two values was taken. The minimal detectable concentrations were $15 \mathrm{pg} / \mathrm{ml}$ VEGF, $0.156 \mathrm{ng} / \mathrm{ml} \pi$-GST, and $5 \mathrm{pg} / \mathrm{ml} \mathrm{MCP}-1$.

\section{Statistical Analysis}

The clinical data represented by eight end points (IAP, urine output, serum creatinine, renal RI, urinary $\pi$-GST, urinary MCP- 1 , urinary VEGF, and serum VEGF) were processed using R-statistics software version 3.3.0. Each of the end points was subject to one-way analysis of variance (ANOVA), followed by one-sided $t$-test vs. the control group, which included multiple hypothesis correction for eight comparisons in two time points separately in each of the two treatment groups. It was not unreasonable to assume normal distribution of end point values. The animal data represented by one end point (serum VEGF) was analyzed by a two-way ANOVA (end point vs. duration of IAH, intensity of IAH, and the interaction of the two), followed by Tukey's post hoc test. By default, all statistical tests were carried out at the $5 \%$ significance level. Regression analyses were performed using ordinary least-squares method and Pearson's correlation coefficient $(r)$. Throughout the paper, the average values are reported as mean $\pm \mathrm{SD}$, where $\mathrm{SD}$ is the standard deviation.

\section{RESULTS}

\section{Patients}

The study included 14 patients with uncomplicated gastroschisis and 13 patients with $\mathrm{CDH}$. The demographic data for the patient groups are summarized in Table 1. Surgical treatment was performed on the day of admission. In gastroschisis group, nine patients underwent primary fascial closure and remaining five patients underwent staged closure with a silo. In $\mathrm{CDH}$ group, eight patients underwent thoracoscopic repair using local tissues and remaining five patients who had had diaphragmatic cupola aplasia underwent repair using either local tissues or a synthetic patch.

IAP. In control patients, IAP was $3.1 \pm 1.3 \mathrm{~mm} \mathrm{Hg}$. Upon admission, in gastroschisis patients IAP did not differ from that in control while in $\mathrm{CDH}$ patients it was increased to $6.0 \pm 1.4 \mathrm{~mm} \mathrm{Hg} \quad(P<0.001)$. Surgical repair of the malformation resulted in significant elevation of IAP in both groups (Figure 1a). By POD4, in gastroschisis patients it increased to $22.2 \pm 2.9 \mathrm{~mm} \mathrm{Hg}$ and in $\mathrm{CDH}$ patients to $19.0 \pm 2.5 \mathrm{~mm} \mathrm{Hg}$ (vs. admission, $P<0.001$ for both). By POD10, IAP partially decreased in both groups, reaching $13.4 \pm 1.0$ and $11.4 \pm 1.3 \mathrm{~mm} \mathrm{Hg}$, respectively (vs. POD4, $P<0.001$ for both).

Urine output. In control patients, urine output was $2.7 \pm 0.7 \mathrm{ml} / \mathrm{kg} / \mathrm{h}$. At admission, in gastroschisis patients it 

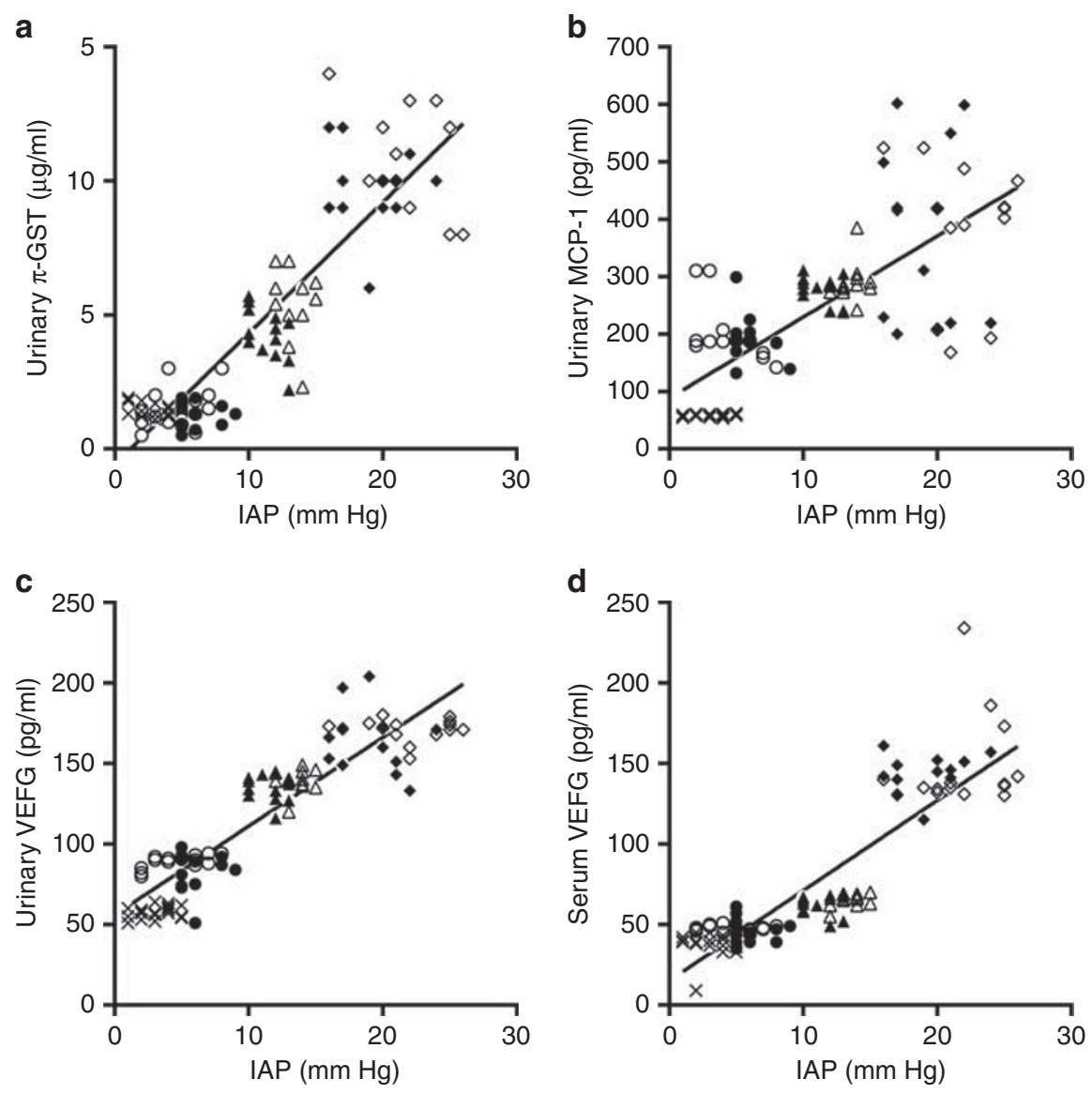

Figure 3. Relationship between intra-abdominal pressure (IAP) and the biomarker levels. (a) Urinary $\pi$-glutathione S-transferase ( $\pi$-GST); (b) urinary monocyte chemoattractant protein-1 (MCP-1); (c) urinary vascular endothelial growth factor (VEGF); (d) serum VEGF. The values are presented as scatter charts. Each symbol corresponds to a single measurement. Crosses denote controls; open symbols denote gastroschisis patients, filled symbols denote congenital diaphragmatic hernia (CDH) patients; circles denote admission values, diamonds denote postoperative day 4 (POD4) values, triangles denote POD10 values. The line represents the optimal least-squares estimate.

was $1.8 \pm 0.6 \mathrm{ml} / \mathrm{kg} / \mathrm{h}$ and in $\mathrm{CDH}$ patients $2.0 \pm 0.5 \mathrm{ml} / \mathrm{kg} / \mathrm{h}$ $(P=0.013$ and $P=0.058$, respectively). By POD4, urine output decreased in both groups, reaching $0.6 \pm 0.3 \mathrm{ml} / \mathrm{kg} / \mathrm{h}$ in gastroschisis and $1.3 \pm 0.2 \mathrm{ml} / \mathrm{kg} / \mathrm{h}$ in $\mathrm{CDH}$ group (vs. admission, $P<0.001$ and $P=0.002$, respectively). By POD10, urine output restored and did not differ from that in control group (Figure 1b).

Creatinine. Upon admission, serum creatinine level in both patient groups did not significantly differ from $43.8 \pm 17.5 \mu \mathrm{mol} / \mathrm{l}$ in control. Correction of the malformation significantly increased the creatinine level by POD4, which reached $113.4 \pm 15.0$ and $93.3 \pm 14.5 \mu \mathrm{mol} / \mathrm{l}$ (vs. admission, $P<0.001$ for both) in gastroschisis and $\mathrm{CDH}$ groups, respectively. By POD10, serum creatinine levels significantly decreased and did not differ from the admission level in any of the patient groups (Figure 1c).

Renal resistive index. In control group, resistive index (RI) was $0.74 \pm 0.02$. Similar admission values of RI were observed in gastroschisis and CDH patients. By POD4, RI significantly increased in both patient groups, reaching $0.83 \pm 0.03$

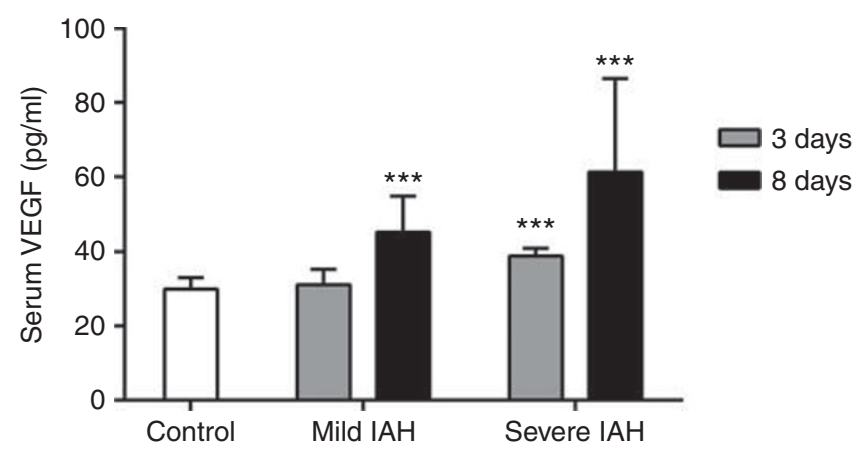

Figure 4. Serum vascular endothelial growth factor (VEGF) levels in newborn rats with intra-abdominal hypertension (IAH). The data are presented as mean $\pm S D$ and analyzed using two-way analysis of variance (ANOVA) (VEGF level vs. IAH intensity, duration, and the interaction of two), followed by Tukey's post hoc test. Comparison vs. control: ${ }^{* * *} P<0.001$.

$(P<0.001)$ and $0.80 \pm 0.04(P=0.009)$, respectively. By POD10, in gastroschisis patients RI was $0.73 \pm 0.05$ and in $\mathrm{CDH}$ patients $0.78 \pm 0.03$; however, the change was not statistically significant (Figure 1d). 


\section{Articles | Morozov et al.}
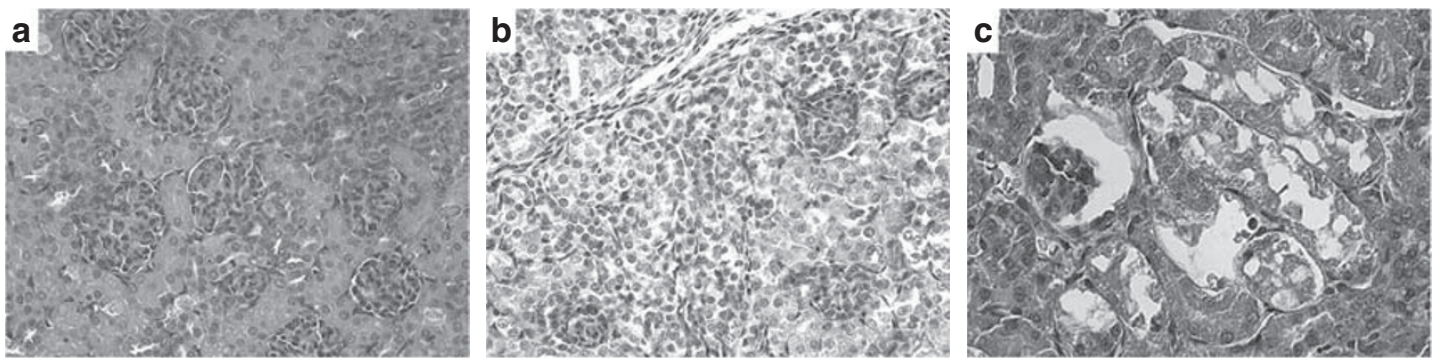

Figure 5. Histopathological changes of the kidney tissue in the model of abdominal compartment syndrome (ACS) in newborn rats. (a) Control; (b) mild intra-abdominal hypertension (IAH) ( $9 \mathrm{~mm} \mathrm{Hg}$ IAP for 8 days); (c) severe IAH (17 mm Hg IAP for 8 days). Hematoxylin and eosin staining; original magnification, $\times 400$.

\section{Biomarkers}

$\boldsymbol{\pi}$-GST. Upon admission, the urinary levels of $\pi$-GST in both patient groups were close to $1.5 \pm 0.2 \mu \mathrm{g} / \mathrm{ml}$ in control. By POD4, in gastroschisis patients the level of $\pi$-GST increased to $11.0 \pm 1.9 \mu \mathrm{g} / \mathrm{ml}$ and in $\mathrm{CDH}$ patients to $9.7 \pm 1.5 \mu \mathrm{g} / \mathrm{ml}$ (vs. admission, $P<0.001$ for both) (Figure 2a). By POD10, the $\pi$ GST levels decreased to $5.5 \pm 1.2$ and $4.3 \pm 1.0 \mu \mathrm{g} / \mathrm{ml}$, respectively (vs. POD4, $P<0.001$ for both), although they were still higher than before the surgery (vs. $P<0.001$ ).

MCP-1. The admission levels of urinary MCP-1 in gastroschisis and $\mathrm{CDH}$ patients were greater than in control: $199 \pm 49$ and $192 \pm 41 \mathrm{pg} / \mathrm{ml}$, respectively, vs. $58 \pm 3 \mathrm{pg} / \mathrm{ml}$ $(P<0.001$ for both) (Figure 2b). By POD4, the MCP-1 levels further increased, reaching $388 \pm 117$ and $376 \pm 154 \mathrm{pg} / \mathrm{ml}$ (vs. admission, $P<0.001$ for both), respectively. By POD10, the levels of MCP-1 decreased to $289 \pm 31 \mathrm{pg} / \mathrm{ml}$ in gastroschisis patients (vs. POD4, $P=0.031$ ) and to $278 \pm 24 \mathrm{pg} / \mathrm{ml}$ in $\mathrm{CDH}$ patients (although the change was not statistically significant, $P=0.114)$.

VEGF. At admission, the urinary VEGF levels in gastroschisis and $\mathrm{CDH}$ patients were greater than in control: $89 \pm 4$ and $82 \pm 12 \mathrm{pg} / \mathrm{ml}$, respectively, vs. $58 \pm 4 \mathrm{pg} / \mathrm{ml} \quad(P<0.001$ for both) (Figure 2c). By POD4, the urinary VEGF levels further increased in both groups, reaching $171 \pm 7$ and $165 \pm 20 \mathrm{pg} / \mathrm{ml}$ (vs. admission, $P<0.001$ for both), respectively. By POD10, the urinary VEGF levels decreased in gastroschisis patients to $139 \pm 7 \mathrm{pg} / \mathrm{ml}$ and in $\mathrm{CDH}$ patients to $135 \pm 8 \mathrm{pg} / \mathrm{ml}$ (vs. POD4, $P<0.001$ for both), while they were still higher than before the surgical treatment. Similar changes were detected for serum VEGF levels in both patient groups (Figure 2d).

Correlation between IAP and the biomarkers. To investigate the relationship between IAP and the biomarker levels, we performed a simple linear regression analysis. The analysis showed that elevation of IAP statistically significantly predicted the increase in the biomarker level (Figure 3). There was a strong positive correlation between the levels of IAP and the levels of serum and urinary VEGF $(r=0.89$ and $r=0.91$, respectively) and urinary $\pi$-GST $(r=0.91)$, followed by urinary MCP-1 levels $(r=0.75)(P<0.001$ for all).

\section{Model of ACS in Newborn Rats}

In the previous part of the study, we demonstrated that urinary and serum levels of the biomarkers differ in newborns with different severity of IAH and strongly correlate with the levels of IAP. To verify whether the changes in the biomarker concentrations were associated with kidney injury caused by elevated IAP, we created a model of IAH in newborn rats. In this model, rat pups were exposed to either mild $(9 \pm 2 \mathrm{~mm} \mathrm{Hg})$ or severe $(17 \pm 2 \mathrm{~mm} \mathrm{Hg})$ IAH for either 3 or 8 days and tested for serum VEGF levels and kidney morphological alterations.

\section{VEGF}

In control rats, serum VEGF level was $30 \pm 3 \mathrm{pg} / \mathrm{ml}$. In a group of mild IAH, VEGF did not change by day 3 of exposure but rose to $45 \pm 9 \mathrm{pg} / \mathrm{ml}$ by day $8 \quad(P<0.001)$ (Figure 4). In a group of severe IAH, VEGF increased to $39 \pm 2$ and $61 \pm 25 \mathrm{pg} / \mathrm{ml}$ by day 3 and day 8 of exposure $(P<0.001$ for both), respectively. Further analysis showed that severity and duration of IAH were independently associated with increased VEGF levels $(P<0.001$ for both), without statistically significant interaction between the two factors $(P=0.136)$.

\section{Renal histology}

In the control group, light microscopy revealed normal histological structure of the renal cortex and medulla (Figure 5a). Induction of mild IAH for 8 days led to a minimal dilation of the glomerular urinary space, mild vacuolization of the proximal tubule epithelial cells, and dilation of the collecting ducts with some epithelial desquamation (Figure 5b). Induction of severe IAH for 8 days led to further dilation of the glomerular urinary space, hydropic degeneration of the epithelium of the proximal tubules, and marked cystic transformation of the proximal tubules and collecting ducts (Figure 5c).

\section{DISCUSSION}

Surgical repair of gastroschisis and $\mathrm{CDH}$ is accompanied by a considerable rise in IAP and a decline in abdominal perfusion pressure. Kidneys are the most sensitive to reduced blood supply and get damaged first (14). In the present study, we investigated the urinary levels of VEGF, $\pi$-GST, and MCP-1 
and serum levels of VEGF as potential biomarkers of renal hypoxic injury in newborns treated for gastroschisis and $\mathrm{CDH}$.

Before surgery, gastroschisis patients had normal and $\mathrm{CDH}$ patients slightly elevated IAP. All the patients demonstrated normal routine laboratory tests and the $\pi$-GST levels, indicating preserved renal function, but showed moderately increased VEGF and MCP-1 levels. This is in line with increased serum levels of VEGF and MCP-1 and their upregulated expression in the pulmonary vascular endothelial cells affected by persistent pulmonary hypertension in $\mathrm{CDH}$ patients. Increased VEGF levels reflect an attempt by the fetus/newborn to enlarge the pulmonary vascular bed in the hypoplastic lungs to alleviate pulmonary hypertension (15), while MCP-1 contributes to recruitment and activation of monocytes, which promote inflammation and pulmonary fibrosis (16). Increased MCP-1 levels were also reported in the cord blood of gastroschisis patients as a result of chronic inflammation in the intestines exposed to amniotic fluid during development (17). Our findings are also consistent with the reports demonstrating leukocyte infiltration in the intestinal wall and increased levels of proinflammatory cytokines in the amniotic fluid in gastroschisis patients (18). Therefore, the changes in the cytokine profile at admission could be regarded as a protective or adaptive response of a newborn to the congenital birth defect.

Reduction of the abdominal viscera and closure of the abdominal cavity caused a significant increase in IAP, as detected on the fourth day after surgery in both patient groups. It was accompanied by increased RI, reduced urine output, and accumulation of serum creatinine, indicating renal hypoperfusion and reduced excretory function. Increase in IAP was also associated with a substantial rise in the urinary VEGF, $\pi$-GST, and MCP-1 levels and serum VEGF levels. $\pi$-GST is a constitutive cytoplasmic enzyme of the distal tubule epithelial cells. It is released into urine following distal tubule damage and serves as a sensitive and specific marker of kidney hypoperfusion and injury (19-21). MCP-1 is a proinflammatory chemokine expressed in proximal tubular epithelial and other kidney cells following ischemic injury $(22,23)$. It plays a major role in recruitment and activation of monocytes and macrophages in the damaged kidney, promoting further tissue destruction $(24,25)$. VEGF is a potent angiogenic factor which is markedly upregulated following renal ischemia in tubular epithelial cells. By stimulating endothelial cell proliferation and neovascularization, it serves for adaptation of a kidney to circulatory hypoxia (26). The increase in the urinary and serum levels of the biomarkers indicates the extent of hypoxic renal injury and adaptation of the renal tissue to hypoperfusion resulting from postsurgery increase in IAP.

Ten days after surgical treatment, IAP partially decreased in both patient groups. It was accompanied by mild, although not significant, reduction in RI, suggesting some improvement of renal blood flow at least in part of our patients, as well as by complete restoration of urine output and creatinine levels, indicating normal excretory function. In contrast, the VEGF, $\pi$-GST, and MCP-1 levels remained significantly elevated. Moreover, we demonstrated that a change in IAP accurately predicted a change in the VEGF and $\pi$-GST levels, pointing out close relationship between IAP and hypoxic renal injury. Although elevation of the urinary VEGF, $\pi$-GST, and MCP-1 levels does not affect the postoperative management, it suggests the ongoing renal injury and inflammation, which put the patients at risk for renal scarring and chronic kidney disease in the follow-up (7-9). Therefore, monitoring for biomarkers predicting the onset of renal scarring would be beneficial in this clinical setting.

To confirm that the changes in the biomarker levels were specifically associated with renal injury that had resulted from sustained elevation of IAP, we created a model of ACS in newborn rats. By using this model, we demonstrated that increase in serum VEGF levels paralleled progressing kidney morphological alteration caused by experimental IAH. Similar histopathological changes have been also shown using alternative models of ACS in adult rats $(27,28)$. These observations support our hypothesis that increased serum and urinary levels of the biomarkers reflect the extent of hypoxic renal injury following surgical treatment for gastroschisis and $\mathrm{CDH}$.

In the present study, the levels of VEGF, $\pi$-GST, and MCP-1 were investigated in patients with two different types of congenital malformations who underwent different surgical treatment. Despite this variability, similar changes in the biomarker levels were revealed in both patient groups, suggesting the causative role for IAH rather than for specific malformation or treatment procedure. The association between the biomarkers levels and morphological alterations in the kidney was demonstrated using the original model of ACS which has several advantages over the other existing models. First, in contrast to the models based on intraperitoneal insufflation of a gas and requiring prolonged general anesthesia (29,30), our model requires only transient anesthesia for collagen injection and IAP measurement and, therefore, is not limited by duration of anesthesia. Second, collagen is stable and not absorbable bulking material allowing to create a sustained IAH lasting for several days without additional manipulations. This cannot be reached in the models based on intraperitoneal instillation of highly absorbable crystalloid solutions $(31,32)$. Furthermore, compared to the model in which IAH is induced by applying an abdominal restraint device (33), our model is closer to the pathophysiology of IAH associated with visceral-abdominal disproportion. We assume that our experimental model of ACS could be useful for the investigation of renal dysfunction not only in newborns treated for gastroschisis or $\mathrm{CDH}$ but also in children receiving a disproportionately large kidney from adult donor and other similar clinical settings.

In conclusion, the major finding of the present study is that urinary VEGF, $\pi$-GST, and MCP-1 levels and serum VEGF levels paralleled the severity of IAH more precisely than the values of urine output and creatinine levels following surgical 


\section{Articles $\mid$ Morozov et al.}

treatment of the malformation in gastroschisis and $\mathrm{CDH}$ patients. By using the original model of ACS in newborn rats, we confirmed that the increase in serum VEGF levels was associated with the extent of kidney morphological alterations resulting from sustained elevation of IAP. Additionally, prolonged elevation of the biomarker levels suggests the ongoing renal injury in newborns with ACS despite complete restoration of renal excretory function several days after surgery. Therefore, a follow-up measurement of VEGF, $\pi$ GST, and MCP-1 levels may provide a better tool for noninvasive assessment of kidney injury and may aid in elaboration of personalized treatment strategy in newborns with ACS.

\section{STATEMENT OF FINANCIAL SUPPORT}

No extramural financial support was received for the present study.

Disclosure: The authors declare no conflict of interest.

\section{REFERENCES}

1. Malbrain ML, Cheatham ML, Kirkpatrick A, et al. Results from the international conference of experts on intra-abdominal hypertension and abdominal compartment syndrome. I. Definitions. Intens Care Med 2006;32:1722-32.

2. Kirkpatrick AW, Roberts DJ, Jaeschke R, et al. Methodological background and strategy for the 2012-2013 updated consensus definitions and clinical practice guidelines from the abdominal compartment society. Anaesthesiol Intens Ther 2015;47 Spec No:s63-77.

3. Kirkpatrick AW, De Waele JJ, De Laet I, et al. WSACS-The Abdominal Compartment Society. A Society dedicated to the study of the physiology and pathophysiology of the abdominal compartment and its interactions with all organ systems. Anaesthesiol Intens Ther 2015;47:191-4.

4. De Waele JJ, Ejike JC, Leppäniemi A, et al. Intra-abdominal hypertension and abdominal compartment syndrome in pancreatitis, paediatrics, and trauma. Anaesthesiol Intens Ther 2015;47:219-27.

5. Divarci E, Karapinar B, Yalaz M, Ergun O, Celik A. Incidence and prognosis of intraabdominal hypertension and abdominal compartment syndrome in children. J Pediatr Surg 2016;51:503-7.

6. Newcombe J, Mathur M, Ejike JC. Abdominal compartment syndrome in children. Crit Care Nurse 2012;32:51-61.

7. Goldstein SL. Acute kidney injury in children and its potential consequences in adulthood. Blood Purif 2012;33:131-7.

8. Harambat J, van Stralen KJ, Kim JJ, Tizard EJ. Epidemiology of chronic kidney disease in children. Pediatr Nephrol 2012;27:363-73.

9. Coca SG, Singanamala S, Parikh CR. Chronic kidney disease after acute kidney injury: a systematic review and meta-analysis. Kidney Int 2012;81:442-8.

10. Mao S, Huang $\mathrm{S}$. The signaling pathway of hypoxia inducible factor and its role in renal diseases. J Recept Signal Transduct Res 2013;33:344-8.

11. de Geus HR, Fortrie G, Betjes MG, van Schaik RH, Groeneveld AB. Time of injury affects urinary biomarker predictive values for acute kidney injury in critically ill, non-septic patients. BMC Nephrol 2013;14:273.

12. Bihorac A, Baslanti TO, Cuenca AG, et al. Acute kidney injury is associated with early cytokine changes after trauma. J Trauma Acute Care Surg 2013;74:1005-13.

13. Abraham MK, Viswanath N, Ramakrishnan P, et al. Intravesical pressure: a new prognostic indicator in congenital diaphragmatic hernia. J Indian Assoc Pediatr Surg 2011;16:129-31.
14. De Waele JJ, De Laet I, Kirkpatrick AW, Hoste E. Intra-abdominal hypertension and abdominal compartment syndrome. Am J Kidney Dis 2011;57:159-69.

15. Shehata SM, Mooi WJ, Okazaki T, El-Banna I, Sharma HS, Tibboel D. Enhanced expression of vascular endothelial growth factor in lungs of newborn infants with congenital diaphragmatic hernia and pulmonary hypertension. Thorax 1999;54:427-31.

16. Okawada M, Kobayashi H, Tei E, Okazaki T, Lane GJ, Yamataka A. Serum monocyte chemotactic protein-1 levels in congenital diaphragmatic hernia. Pediatr Surg Int 2007;23:487-91.

17. Frascoli M, Jeanty C, Fleck S, et al. Heightened immune activation in fetuses with gastroschisis may be blocked by targeting IL-5. J Immunol 2016;196:4957-66.

18. Guibourdenche J, Berrebi D, Vuillard E, et al. Biochemical investigations of bowel inflammation in gastroschisis. Pediatr Res 2006;60:565-8.

19. Walshe CM, Odejayi F, Ng S, Marsh B. Urinary glutathione S-transferase as an early marker for renal dysfunction in patients admitted to intensive care with sepsis. Crit Care Resusc 2009;11:204-9.

20. Ralib AM, Pickering JW, Shaw GM, Than MP, George PM, Endre ZH. The clinical utility window for acute kidney injury biomarkers in the critically ill. Crit Care 2014;18:601.

21. Shu $\mathrm{KH}$, Wang $\mathrm{CH}, \mathrm{Wu} \mathrm{CH}$, et al. Urinary pi-glutathione $S$-transferase predicts advanced acute kidney injury following cardiovascular surgery. Sci Rep 2016;6:26335.

22. Segerer S, Nelson PJ, Schlondorff D. Chemokines chemokine receptors, and renal disease: from basic science to pathophysiologic and therapeutic studies. J Am Soc Nephrol 2000;11:152-76.

23. Rice JC, Spence JS, Yetman DL, Safirstein RL. Monocyte chemoattractant protein-1 expression correlates with monocyte infiltration in the postischemic kidney. Ren Fail 2002;24:703-23.

24. Stroo I, Claessen N, Teske GJ, Butter LM, Florquin S, Leemans JC. Deficiency for the chemokine monocyte chemoattractant protein-1 aggravates tubular damage after renal ischemia/reperfusion injury. PLoS ONE 2015;10:e0123203.

25. Furuichi K, Wada T, Iwata $\mathrm{Y}$, et al. Gene therapy expressing aminoterminal truncated monocyte chemoattractant protein-1 prevents renal ischemia-reperfusion injury. J Am Soc Nephrol 2003;14:1066-71.

26. Liu F, Lou YL, Wu J, et al. Upregulation of microRNA-210 regulates renal angiogenesis mediated by activation of VEGF signaling pathway under ischemia/perfusion injury in vivo and in vitro. Kidney Blood Press Res 2012;35:182-91.

27. Akbulut G, Altindis M, Aktepe F, Serteser M, Dilek ON. Renal cytokine and histopathologic changes following acutely increased intra-abdominal pressure: an animal study. Ulus Travma Acil Cerrahi Derg 2010;16:103-7.

28. Kosum A, Borazan E, Maralcan G, Aytekin A. Biochemical and histopathological changes of intra-abdominal hypertension on the kidneys: experimental study in rats. Ulus Cerrahi Derg 2013;29:49-53.

29. Sui F, Zheng Y, Li WX, Zhou JL. Renal circulation and microcirculation during intra-abdominal hypertension in a porcine model. Eur Rev Med Pharmacol Sci 2016;20:452-61.

30. Akkapulu N, Tirnaksiz MB, Kulac I, et al. Effects of intra-abdominal pressure on adrenal gland function and morphology in rats. Int J Clin Exp Pathol 2015;8:3230-7.

31. Meier C, Contaldo C, Schramm R, et al. A new model for the study of the abdominal compartment syndrome in rats. J Surg Res 2007;139: 209-16.

32. Yoshino O, Quail A, Oldmeadow C, Balogh ZJ. The interpretation of intra-abdominal pressures from animal models: the rabbit to human example. Injury 2012;43:169-73.

33. Chang M, Yu J, Zhang L, et al. A new model for the study of secondary intra-abdominal hypertension in rats. J Surg Res 2014;187:244-51. 